\title{
A robust digital method for film contrast correction in subtraction radiography
}

\author{
Urs E. Ruttimann, Richard L. Webber, and Edgar Schmidt \\ Diagnostic Systems Branch, National Institute of Dental Research, NIH Bethesda, Maryland, \\ and The University of Michigan, School of Dentistry, Ann Arbor, USA.
}

\begin{abstract}
Digital subtraction radiography requires close matching of the contrast in the films to be subtracted. A digital method is presented permitting the retrospective correction of film contrast differences. The method is nonparametric and derives the required gray level transformation directly from the histograms associated with the radiographs. This transform is shown to be unique and monotonic. It is based on fewer theoretical assumptions than a previously described parametric correction method, and it performs significantly better in reducing the contrast mismatch measured by the standard deviation of the gray levels in the subtraction image.
\end{abstract}

Accepted for publication December 5, 1985

\section{Introduction}

Subtraction radiography (Grondahl, Grondahl \& Webber 1983 a,b) is a potentially powerful method for monitoring the results of periodontal therapy. Its successful application in longitudinal studies requires that the variations caused by uncontrolled changes of the x-ray imaging geometry, as well as by film exposure and processing conditions, be small relative to the changes of diagnostic interest occurring over the observation interval. While these prerequisites are difficult to attain, controlled studies (Rosling et al. 1983, Rethman et al. 1985) have demonstrated that geometrical registrations using occlusal templates (Rosling et al. 1975, Duckworth et al. 1983) result in detectability of periodontal lesions that is significantly improved over conventional radiographic methods. Reproducibility of film contrast can be maintained by electronic control of exposure time, $x$-ray tube current and voltage waveforms, and by careful film processing with quality control of the chemicals. Despite these efforts, contrast discrepancies do occur in practice. This report presents a new digital method for correcting retrospectively film-contrast differences in radiographs to be subtracted, and compares it to a technique published previously (Ruttimann et al. 1981).

\section{Material and Methods}

The radiographs were obtained from an ongoing double-blind clinical investigation at the University of Michigan, which is testing the efficacy of metronidazole in the treatment of periodontitis. The patients admitted to the study had clinical evidence of moderate to advanced periodontitis. Utilized for this study were 12 pairs of radiographs taken at 2 different occasions in the protocol, either prior to any treatment, after the hygiene phase, or after periodontal sur- 
gery. Geometrical registration of the radiographs was obtained using occlusal registration/film holder devices (Duckworth et al. 1983). The film holders were coupled mechanically to the cone of the $x$-ray machine and the films* were exposed for $.3 \mathrm{~s}$ (posterior teeth) or $.24 \mathrm{~s}$ (anterior teeth) at $70 \mathrm{kVp}$ and $15 \mathrm{~mA}$. The exposed films were developed in an automated developer $\dagger$ at 30 C. Subtraction images of the radiographs were obtained by digital subtraction of corresponding $512 \times 512 \times 8$ bit video frames using a computer-interfaced image processor\$ as described in (Grondahl et al. 1983a).

The digital images were corrected for variations in contrast prior to subtraction by applying separately both a previously described parametric method and the newly developed nonparametric method described in detail below. The parametric contrast correction procedure (Ruttimann et al. 1981) is based on matching the first 2 moments of the gray level distributions in the radiographic images to be subtracted. This is achieved by employing a quadratic transform $y=a x^{2}+b x+c$ mapping the gray levels $\mathrm{x}$ in image 1 into the gray levels $\mathrm{y}$ in image 2 (Fig. 1). The coefficients $a, b$, and $\mathrm{c}$ are determined by 3 equations requiring that the highest possible gray levels $\mathrm{gl}_{\mathrm{H}}$, in both images map into each other, and that mean $\mu_{1}$ and standard deviation $\sigma_{1}$ transform into the corresponding parameters $\mu_{2}$ and $\sigma_{2}$ of the gray level distribution in the second image. Hence, the name parametric method was given.

The new contrast correction method proposed here is nonparametric and does not rely on matching parameters of correspond-

* Periapical, Ektaspeed, Eastman Kodak Co., Rochester, NY 14650

$\uparrow$ Philips 810, Philips Medical Systems, Inc., Shelton, CT 06484

‡ IP 6400, Gould Inc., DeAnza Imaging \& Graphics Division, San Jose, CA 95131

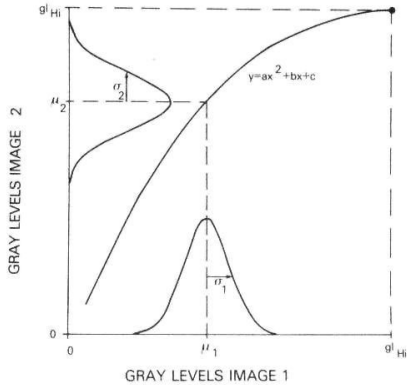

Fig. 1. Parametric correction method gray-level transformation $y$ is based on the means $\mu_{1}, \mu_{2}$, the standard deviations $\sigma_{1}$ and $\sigma_{2}$, and the highest possible gray level $\mathrm{g}_{\mathrm{H}_{\mathrm{i}}}$ in the images

ing distributions. Its principle is outlined here only, a rigorous derivation is presented in the Appendix. Let us assume that the quantized gray scale in the images ranges from $1-N$. Then for the image with the histogram of the desired shape, let the number of picture elements (pixels) with gray level $i$ be denoted by $p_{i}$, forming the $p$-histogram. Similarly, for the image with the histogram to be modified, $q_{j}$ contains the count of pixels with gray level $\mathrm{j}$, forming the q-histogram. We define for the p-histogram the sum over the counts $p_{i}$ from gray level 1 up to gray level $\mathrm{k}$ by $\mathrm{S}_{\mathrm{k}}$, and analogously for the $\mathrm{q}$-histogram, the sum over the counts $\mathrm{q}_{\mathrm{i}}$ from 1 up to gray level $m$ by $T_{m}$. It is then required that these cumulative sums be closely matched at every gray level $\mathrm{m}$ as derived in the Appendix:

$$
\max _{k}\left[\mathrm{~T}_{\mathrm{m}-1}<\mathrm{S}_{\mathrm{k}} \leq \mathrm{T}_{\mathrm{m}}\right],
$$

where $\mathrm{m}=1,2, \ldots, \mathrm{N}$, and $\mathrm{T}_{0}=0$. This means that we find for each gray level $\mathrm{m}$ in the histogram to be modified (q-histogram) the largest gray level $\mathrm{k}$ in the target (p-histogram) such that the cumulative sum over 
the p-histogram up to $\mathrm{k}$ is just equal to or smaller than the cumulative sum over the $\mathrm{q}$-histogram up to $\mathrm{m}$, but strictly larger than the value of that sum up to $m-1$. This correspondence between $\mathrm{k}$ and $\mathrm{m}$ defines the gray level transform required. For example, assume that a correspondence has been found between $\mathrm{m}=3$ and $\mathrm{k}=5$, and similarly between $m=7$ and $k=6$. Then the gray levels in neighboring bins from $\mathrm{m}=4$ up to and including $m=7$ require pooling to match the cumulative sum $\mathrm{S}_{k}$ at $\mathrm{k}=6$; i.e. gray levels $4,5,6$, and 7 of the q-histogram are mapped into gray level 6 of the phistogram. This transformation only requires pooling; and splitting of gray levels in the histogram to be modified into two or more different gray levels in the p-histogram does not occur.

It is shown in the Appendix that the graylevel mapping defined above is both unique and order-preserving, i.e., the larger of any pair of gray levels before modification remains after the transform larger than, or is at least equal to the transformed value of the smaller gray level.

Fig. 2 demonstrates the principle of this histogram modification process for images with only 8 gray levels (Row 1), and the simple case of a uniformly distributed target histogram; i.e., for all 8 gray levels $p_{i}=16$ (Row 2). The third row shows the successive values of $S_{k}$, the cumulative sum over the values of $\mathrm{p}_{i}: \mathrm{S}_{1}=16, \mathrm{~S}_{2}=16+16=32, \mathrm{~S}_{3}=$ $32+16$, etc. The bottom row labelled $\mathrm{q}_{j}$ displays the histogram to be modified, and the row above it shows the sequence of the

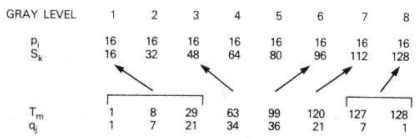

Fig. 2. Principle of histogram modification for images with 8 gray levels.

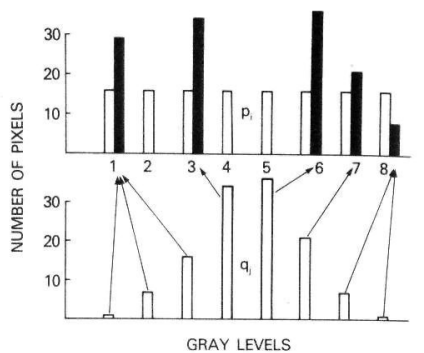

Fig. 3. Example from Fig. 2. Bottom: histogram to be modified. Top: target histogram (open bars), and histogram after modification (filled bars).

corresponding cumulative sums $\mathrm{T}_{m}: \mathrm{T}_{1}=1$, $\mathrm{T}_{2}=1+7=8, \mathrm{~T}_{3}=8+21=29$, etc. It is seen that the first 3 gray levels in the q-histogram with $\mathrm{q}_{j}=1,7$, and 21 must be pooled in order to satisfy the inequality condition for $\mathrm{S}_{1}: \mathrm{T}_{2}=8<\mathrm{S}_{1}=16 \leq \mathrm{T}_{3}=29$. For the next gray level in the q-histogram with $\mathrm{T}_{4}=63$, 2 inequality conditions involving $\mathrm{S}_{2}$ and $\mathrm{S}_{3}$ hold: $29<32<63$, and $29<48<63$. According to the definition for the mapping given above, the larger of the two $\mathrm{S}_{k}$ 's is selected for the mapping; i.e., gray level 4 of the q-histogram is mapped into gray level 3 , as indicated by the arrow. Further successive evaluation of the inequality conditions for each of the remaining $T_{m}$ completes the construction of the gray level transformation as indicated by the arrows in Fig. 2.

Fig. 3 shows the result of this histogramrebinning process for the example computed above. The open bars display the target histogram on the top, and below the histogram to be modified. The filled bars represent the histogram obtained after transformation. While perfect uniformity cannot be achieved in this simple case with only 8 different gray levels, the resulting 
histogram certainly displays a more uniform distribution than the original shown beneath it. Furthermore, it is to be noted that because some bins of the original histogram were pooled in the rebinning process, certain gray levels are no longer present in the image after the modification; i.e., gray levels 2,4 , and 5 are missing. This is due to a general property of the mapping process as defined above, which permits only pooling of neighboring gray level bins, but not splitting of one gray level into several others.

\section{Results}

Fig. 4 shows a pair of radiographs to be subtracted. The film on the right was underexposed and, thus, had a higher mean gray level $\mu=170.58$, and a lower contrast as indicated by the standard deviation $\sigma=$ 29.93 than the film on the left with $\mu=$ 122.51 and $\sigma=34.67$.

The bottom row of Fig. 5 displays the respective gray level histograms of the 2 radiographs. As reflected in the corresponding means and standard deviations, the histogram of the underexposed radiograph is displaced to the right, and narrower compared to the histogram of the properly exposed film. The curves labelled integral in the 2 graphs represent the respective cumulative sums $S_{k}$ and $T_{m}$ to be matched by the modification process. The graph on the top right shows the transfer function obtained by the matching procedure. It maps the gray levels in image 1 associated with the histogram beneath into the gray levels in image 2 , producing the modified histogram on the top left. This histogram is to be compared with its target shown below it. The resulting mean and standard deviation are $\mu=122.48$ and $\sigma=34.73$, confirming the global match obtained by a general shift to the left and a slight stretch of the original histogram. Local effects of the modification procedure may be seen by comparing the small side lobes on the left sides of each of the histograms. This side lobe is built up in the re-

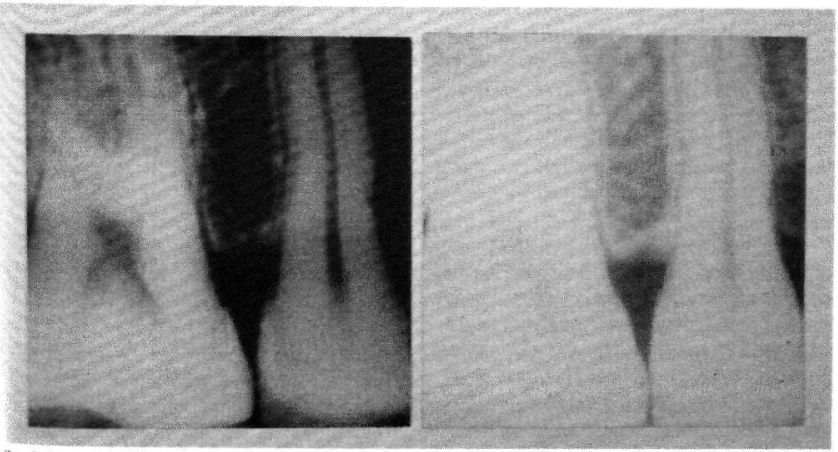

ig. 4. Pair of radiographs to be subtracted. Left: properly exposed film $\mu=122.51, \sigma=34.67$, right: underexposed Im, $\mu=170.58, \sigma=29.93$. 

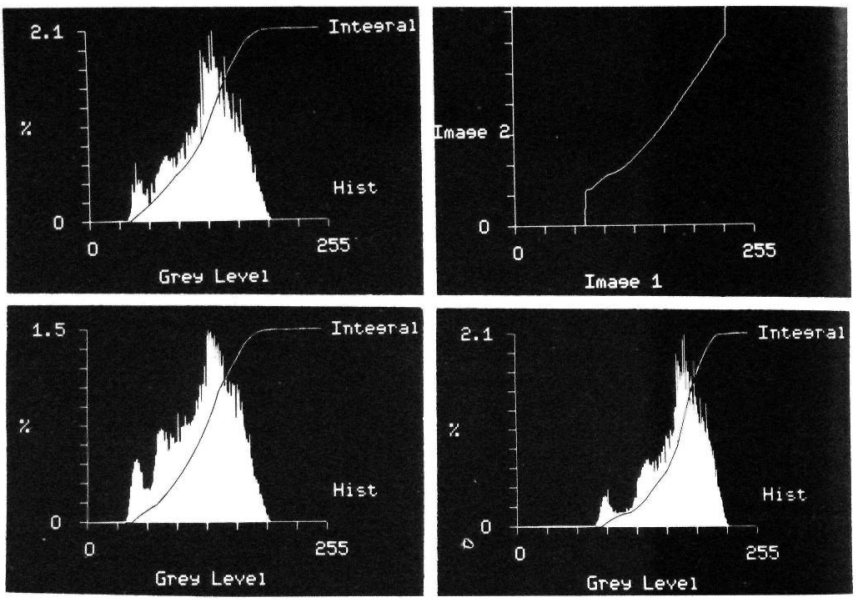

Fig. 5. Bottom: histograms of properly exposed (left) and underexposed (right) films. Top: histogram after the modification using gray-level transfer characteristic shown to the right. Curves labelled integral represent the cumulative sums.

sulting histogram and the gap between the main lobe narrowed compared to the original histogram, in order to obtain a closer match to the shape of the target. This local shape matching property of the nonparametric method constitutes a significant advantage over the parametric method, which can only achieve a global matching of the histograms.

Fig. 6 compares the resulting subtraction images using either of the methods. Subtraction of the unmatched radiographs is shown on the left. Clearly, unacceptably large graylevel differences exist in the uncorrected subtraction image between the areas associated with dentin, bone and soft tissue. Contrast matching by the parametric method yields a markedly improved subtraction image, shown in the center. However, the root canal in the bicuspid, and a hint of the trabecular pattern in the alveolar bone are still visible. The subtraction after contrast matching by the nonparametric method shown on the right results in the most uniform image with the least amount of visible structures present. Note in particular, that compared to the subtraction after parametric matching, the soft tissue region is better matched to the adjacent areas representing dentin and alveolar bone. This region occupies gray levels characterized by the small side lobe on the left side in each histogram, as discussed in Fig. 5. Shown below each of the subtraction images arc the associated histograms, confirming the results of the visual inspection. The globa mismatch of the histograms in the origina radiographs resulted in a displacement of 


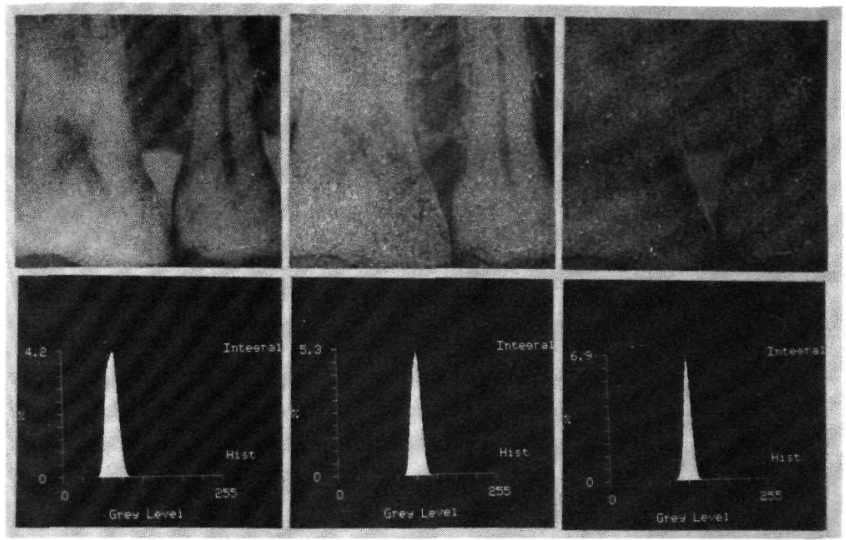

Fig. 6. Top row: Subtraction images, bottom row: corresponding histograms. Left: uncorrected radiographs $\mu=$ 78.93, $\sigma=9.78$. Middle: radiographs corrected by parametric method, $\mu=127.31, \sigma=7.35$. Right: radiographs corrected by non-parametric method, $\mu=127.03, \sigma=6.50$

the mean gray level in the subtraction image from the central value of 127 to $\mu=78.93$. and in a standard deviation of $\sigma=9.78$. Both parametric and nonparametric corrections resulted in good mean gray level matches with $\mu=127.31$ and 127.03 , respectively, and in standard deviations ordered according to their potential for local histogram matching with $\sigma=7.35$ and 6.50 respectively.

A further example of the relative performance of the 2 methods is demonstrated in Fig. 7. The top row shows a pair of original radiographs with significant contrast differences. At the bottom left is the subtraction image after parametric correction, and on the right after nonparametric matching. The relatively low gray level associated with the regions of soft tissue in the left image may leave some uncertainty about the extent of the crestal bone loss indicated by the dark band. The local histogram matching achieved by the nonparametric method resulted in a better correction of the gray levels associated with soft tissue (arrow), and, thus, the bone loss is displayed with a higher contrast.

The parametric and the nonparametric matching methods were each applied to 12 pairs of radiographs obtained from an ongoing clinical study. The standard deviations in the gray level histograms of the resulting subtraction images were taken as the performance measure for comparing the methods. The graph in Fig. 8 summarizes the results, with the lengths of the bars depicting the observed standard deviations. Except for one case, the standard deviations using the nonparametric method were lower, yielding statistical significance $(p<.01)$ of the difference by the paired Wilcoxon test. 


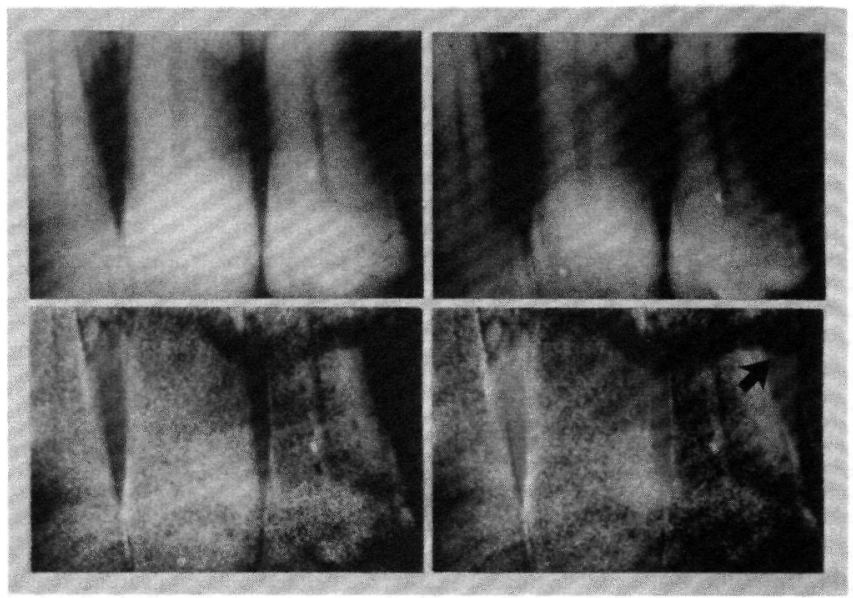

Fig. 7. Top row: Pair of radiographs to be subtracted. Bottom row: subtraction image after parametric correction (left), $\sigma=15.15$, and after nonparametric correction (right), $\sigma=13.86$. Arrow points out improved detail contrast indicating bone loss.

\section{Discussion}

The parametric method has been shown to work well in experimental (Ruttimann et al. 1981, Rethman et al. 1985) and clinical (Rosling et al. 1983) subtraction radiography. For theoretical reasons, the parametric method is limited to, and thus, expected to work best for, "smooth" histograms that are relatively well concentrated around their mean gray levels. When the histograms arise from Gaussian distributions this method is, of course, exact. However, the presence of multiple strong peaks and/or large skew in one of the histograms violates the mathematical assumptions underlying the parametric method, and, thus, suboptimal results or even occasional gross errors must be expected under these circumstances. In

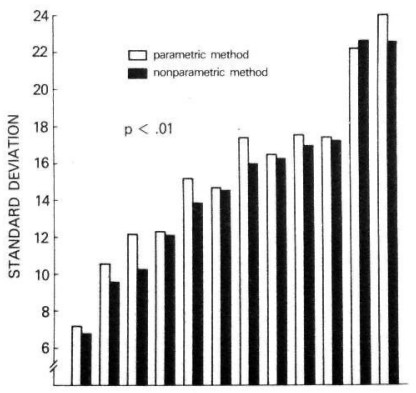

SUBTRACTION IMAGES

Fig. 8. Standard deviations in 12 subtraction images after parametric (open bars) and nonparametric correction (filled bars). 
order to alleviate possible problems, a new, nomparametric method was proposed that does not rely on matching parameters of smooth distributions approximating the observed histograms. Instead, the proposed method operates directly on the histograms themselves. It permits modifying the shape of a histogram associated with a given image into any other desired shape, without posing any restricting assumptions regarding the particular shapes of the respective histograms involved. In particular, the histograms may be multimodal, and separate matching of the individual modes can be achieved. The latter property was referred to above as local histogram matching capability.

The gray-level mapping procedure is based on shifting and/or pooling bins associated with the histogram to be modified such that the cumulative sum over the counts in its bins extending from one up to each gray level is matched as closely as possible to the corresponding sum in the target histogram, without splitting existing bins into different gray levels. This modification principle is rigorous mathematically and has been proven to generate a graylevel mapping which is both unique and monotonic. Uniqueness means that for each gray level in the original histogram there is only one possible gray level in the target into which it can be mapped. Monotonicity implies that the transform cannot introduce gray-level reversals into a sequence of, say, monotonically increasing gray levels taken from a string of neighboring pixels in the image to be modified. Without this assurance the transform could generate false contours in the modified image. Monotonicity is demonstrated in the upper right graph of Fig. 5, showing that for any increase of the gray level in image 1 the curve representing the transfer function either increases, or at ieast stays at the same level.

While both contrast correction proce- dures achieve global matching of the histograms concerned, i.e., the respective means and standard deviations are sought to be equalized, only the nonparametric method has a local matching capability. The importance of this property is demonstrated in Figs. 6 and 7 by the superior matching of the gray levels associated with soft tissue areas in the radiographs. These gray levels are generally located in the left tails of the histograms, and may at times display strong modes. Proper matching of side lobes in the histograms is important for achieving a background as uniform as possible in the subtraction image, in order to better discern evidence of true tissue changes.

The standard deviations of the gray levels in the subtraction images are a function of both the global and local histogram matching attained. Although it is theoretically possible that one matching could be traded for the other while maintaining a constant standard deviation, it is highly unlikely that this will happen with the proposed nonparametric method for images of practical concern. Hence, the reduction in standard deviation attained by the nonparametric method may be considered largely the result of the local histogram matching achieved. Also, the standard deviations may not fully reflect the often substantial visual improvements achieved by local histogram matching. With due consideration of these limitations of the standard deviation as a means for comparing the methods, the results shown in Fig. 8 clearly demonstrate superiority of the nonparametric procedure. Hence, smaller standard deviations can be expected to be attained in the majority of subtractions of clinically obtained radiographs. These smaller standard deviations signify the better gray level matchings achieved for the respective areas of enamel, bone, dentin, and soft tissue, enhancing the chance for the detection of more subtle anatomical changes. 
In conclusion, the nonparametric method is based on fewer theoretical assumptions and, thus, is more robust in practical applications than the parametric approach; and it yields statistically significantly better results in terms of standard deviations in the subtraction images, indicating a closer matching of the respective gray levels associated with different anatomical areas in the radiographs.

\section{Appendix}

The digital images are quantized into $\mathrm{N}$ gray levels. Let $p_{i}$ denote the number of pixels with gray level $\mathrm{i}$ in the target histogram (p-histogram), and similarly let $\mathrm{q}_{\mathrm{j}}$ denote the number of pixels with gray level $\mathrm{j}$ in the histogram to be modified (q-histogram). Thus,

$$
p_{i} \geq 0, q_{i} \geq 0 ; i, j=1,2, \ldots, N .
$$

The cumulative sums over the respective histograms are given by

$$
\mathrm{S}_{k}=\sum_{i=1}^{k} \mathrm{p}_{i}, \mathrm{~T}_{m}=\sum_{j=1}^{m} \mathrm{q}_{\mathrm{i}}, \mathrm{k}, \mathrm{m} \varepsilon[1, \mathrm{~N}] .
$$

From (1) and (2) it follows immediately that $\mathrm{S}_{k}$ and $\mathrm{T}_{m}$ are monotonic increasing functions of the integers $\mathrm{k}$ and $\mathrm{m}$, respectively; i.e., for all gray levels if $k^{\prime}<k^{\prime \prime}$ then $S_{k^{\prime}} \leq S_{k^{\prime \prime}}$ (or if $\mathrm{m}^{\prime}<\mathrm{m}^{\prime \prime}$ then $\mathrm{T}_{m^{\prime}} \leq \mathrm{T}_{m^{\prime \prime}}$ ). We define a mapping of gray level $\mathrm{k}$ from the p-histogram into gray level $m$ of the q-histogram subject to the constraints

$$
\mathrm{T}_{m-1}<\mathrm{S}_{k} \leq \mathrm{T}_{m}, \mathrm{~m}=1,2, \ldots, \mathrm{N}, \mathrm{T}_{0}=0 .
$$

Assertion: For each $\mathrm{k} \varepsilon[1, \mathrm{~N}]$ there exists only one $\mathrm{m} \varepsilon[1, \mathrm{~N}]$ which satisfies (3).

Proof: Assume (3) is satisfied by $m$ and by $\mathrm{n} \neq \mathrm{m}$. Hence,

$$
\begin{aligned}
& \mathrm{T}_{m-1}<\mathrm{S}_{k} \leq \mathrm{T}_{m} \\
& \mathrm{~T}_{n-1}<\mathrm{S}_{k} \leq \mathrm{T}_{n} .
\end{aligned}
$$

From the right side of (5) it follows that

$$
\mathrm{S}_{k}-\mathrm{T}_{n} \leq 0 \text {. }
$$

Subtracting $T_{n}$ from the left side of (4), and using (6) yields

$$
\mathrm{T}_{m-1}-\mathrm{T}_{n}<\mathrm{S}_{k}-\mathrm{T}_{n} \leq 0 .
$$

If $\mathrm{m}>\mathrm{n}$, then $\mathrm{m}-1 \geq \mathrm{n}$, and because $\mathrm{T}_{j}$ is monotonic it follows that $\mathrm{T}_{m-1}-\mathrm{T}_{n} \geq 0$, which is a contradiction to (7). Similarly, if $\mathrm{m}<\mathrm{n}$ it can be shown that $\mathrm{T}_{n-1}-\mathrm{T}_{m} \geq 0$ leads to a contradiction with (4) and (5). Hence, (4) and (5) cannot hold simultaneously, unless $\mathrm{m}=\mathrm{n}$, (Q.E.D.).

Using the same reasoning as in the proof above, it can be shown that the gray level transform defined by (3) preserves the ordering of the gray levels in the 2 histograms, i.e.,

$$
\text { if } \mathrm{k}^{\prime}<\mathrm{k}^{\prime \prime} \text { then } \mathrm{m}^{\prime} \leq \mathrm{m}^{\prime \prime} \text {. }
$$

Note, so far we have shown that for each $\mathrm{k}$ there is only one m satisfying (3). There may, however, exist several gray levels $\mathrm{k}^{\prime}<\mathrm{k}^{\prime \prime}<\ldots$, for which (3) is satisfied by the same value of $m$ as indicated in (8), when equality exists between $\mathrm{m}^{\prime}$ and $\mathrm{m}^{\prime \prime}$. Hence, in order to make the mapping between $\mathrm{k}$ in the $\mathrm{p}$-histogram and $m$ in the $q$-histogram unique, we select the largest possible gray level from among the admissible gray levels $\mathrm{k}^{\prime}<\mathrm{k}^{\prime \prime}<\ldots$ associated with the same $\mathrm{m}$. Consequently, the mapping satisfying the constraints

$$
\begin{aligned}
\max _{k}\left[\mathrm{~T}_{\mathrm{m}-1}<\mathrm{S}_{\mathrm{k}} \leq \mathrm{T}_{\mathrm{m}}\right] & \\
\mathrm{m} & =1,2, \ldots, \mathrm{N}, \mathrm{T}_{0}=0,
\end{aligned}
$$

ensures uniqueness of the relation between the gray levels $\mathrm{k}$ and $\mathrm{m}$.

\section{References}

Duckworth, J. E., Judy, P. F., Goodson, J. M. \& Socransky, S. S. 1983. A method for the geometric and densitometric standardization of intraoral radiographs. Journal of Periodontology 54: $435-440$. 
Grondahl, H.-G., Grondahl, K. \& Webber, R. L. 1983a. A digital subtraction technique for dental radiography. Oral Surgery. Oral Medicine. Oral Pathology 55: 96-102.

Grondahl, H.-G., Grondahl, K. \& Webber, R. L. 1983b. Digital subtraction radiography for diagnosis of periodontal bone lesions with simulated fast speed systems. Oral Surgery, Oral Medicine, Oral Pathology 55: 313-318.

Rethman, M. P., Ruttimann, U. E., O'Neil, R. B., Webber, R. L., Davis, A. A. \& Woodyard, S. G. 1985. Diagnosis of bone lesions by subtraction radiography. Journal of Periodontology 56: $324-329$.

Rosling, B. G., Slots, J., Webber, R. L., Christersson, L. A. \& Genco, R. J. 1983. Microbiological and clinical effects of topical subgingival antimicrobial treatment on human periodontal disease. Journal of Clinical Periodontology 10: 487-514.

Rosling, B. G., Hollender, L., Nyman, S. \&
Olsson, G. 1975. A radiographic method for assessing changes in alveolar bone height following periodontal therapy. Journal of Clinical Periodontology 2: 211-217.

Ruttimann, U. E., Okano, T., Grondahl, H.-G., Grondahl, K. \& Webber, R. L. 1981. Exposure geometry and film contrast differences as bases for incomplete cancellation of irrelevant structures in dental subtraction radiography. Proceedings of the International Society for Optical Engineering (SPIE) 314: 372-377.

\section{Address:}

Dr. Urs E. Ruttimann

Senior Staff Fellow

Diagnostic Systems Branch, NIDR

Building 10, Room 5N-268

9000 Rockville Pike

Bethesda, MD 20892

USA 
This document is a scanned copy of a printed document. No warranty is given about the accuracy of the copy. Users should refer to the original published version of the material. 